\title{
Percepção do Estudante de Medicina sobre a Inserção da Radiologia no Ensino de Graduação com Uso de Metodologias Ativas
}

\author{
Perceptions of a Medical Students on the \\ Inclusion of Radiology the Teaching Degree \\ through the Use of Active Methodologies
}

\author{
Alexandre Ferreira da Silva \\ Robson José de Souza Domingues ${ }^{I}$ \\ Kátia Simone Kietzer ${ }^{1}$ \\ Jofre Jacob da Silva Freitas ${ }^{I}$
}

\section{PALAVRAS-CHAVE}

- Educação Médica.

- Radiologia.

- Aprendizagem Baseada em Problemas.

- Estudantes de Medicina.

- Ensino.

\section{RESUMO}

A radiologia no curso de graduação em Medicina alcançou nova dimensão não só como instrumento de diagnóstico complementar, mas também por sua utilização como ferramenta de ensino, integrada ao estudo da anatomia e patologia, entre outros assuntos. Embora os conhecimentos da radiologia sejam bastante utilizados no ensino da medicina através de metodologias ativas, ainda são escassos os trabalhos relatando a experiência de como o aluno percebe a importância desse conhecimento. Objetivo: Estudar a percepção de alunos de medicina sobre a inserção da radiologia no ensino de graduação com uso de metodologias ativas. Método: Estudo qualitativo, realizado com estudantes de medicina matriculados no terceiro e quinto semestres, cuja amostra constituiu-se de 12 alunos de uma população de 100 alunos de uma universidade pública estadual. Os dados foram obtidos através de entrevistas, com a aplicação de um questionário semiestruturado com questões abertas sobre o ensino da radiologia, suas implicações e aplicações em medicina, características positivas e negativas, no processo de aprendizagem através do uso de metodologias ativas. Os dados foram analisados separadamente, agrupados por categorização e submetidos à análise temática por dois pesquisadores, antes da reflexão conjunta, havendo ainda a descrição do ambiente e também a triangulação dos resultados. Resultados: Foram identificados conteúdos latentes e semânticos, recorrentes, singulares e controversos. Os temas recorrentes identificados como fundamentos de uma estratégia vantajosa para o ensino do radiodiagnóstico na graduação médica foram: necessidade de introdução precoce da radiologia por conta de sua transversalidade e seu papel na atividade de ensino em serviço; ensino sistemático dos princípios biofísicos e vocabulário para facilitar o estudo; o papel do exame complementar no contexto do problema na tutoria e o desenvolvimento de material didático de conteúdo integrado com abordagem morfofuncional. Aspectos singulares como a dificuldade para estudo que envolva conhecimento de lingua estrangeira e o uso de redes sociais, $e$ controvérsias identificadas, a respeito do papel do professor no processo de ensino e aprendizagem, bem como, em relação ao significado do aprendizado da radiologia na graduação em medicina, também foram considerados elementos construtivos de uma abordagem moderna, já que o ensino da radiologia vive um momento de reformulação. Conclusão: Observou-se que na percepção dos estudantes entrevistados, o componente curricular morfofuncional, frequentemente utilizado no ensino baseado em problemas poderá ser usado para abordar a radiologia e diagnóstico por imagem numa visão multidimensional, contextualizada e interdisciplinar que vá além do papel de método de exame complementar, integrado a outros saberes de forma significativa, especialmente anatomia e patologia, facilitando a aprendizagem tanto da radiologia quanto dos demais assuntos envolvidos na temática abordada em cada módulo de estudo. 


\section{KEY-WORDS}

- Medical Education.

- Radiology.

- Problem-Based Learning.

- Medical students.

- Teaching.

Recebido em: 16/9/18

Radiology in medical school has taken on a new dimension in terms of its usefulness, not only as a complementary diagnostic instrument but also as a teaching tool integrated into the study of anatomy and pathology, among other subjects. Although knowledge of radiology is widely used in medical teaching, through active methodologies, there is a lack of studies reporting experiences of how students view the importance of this knowledge. Aim: To study the perceptions of medical students on the inclusion of radiology in undergraduate education, through the use of active methodologies. Method: A qualitative study, conducted with students enrolled in the third and fifth semesters. The sample comprised 12 subjects chosen from a population of 100 students at a public state university. Data were obtained from interviews using a semi-structured questionnaire with open questions about radiology teaching, its implications and applications in medicine, and positive and negative characteristics of the learning process using active methodologies. Data were analyzed separately, grouped by categorization, and submitted to thematic analysis by two researchers, before being reflected on jointly. There was also description of the environment and triangulation of the results. Results: Latent, semantic, recurrent, unique, and controversial contents were identified. The recurrent themes in the interviews, identified as forming the basis of an advantageous strategy for radiodiagnostic teaching in undergraduate medical education, were as follows: (a) the need of an early introduction to radiology, due to its transversality and its importance to teaching; (b) a systematic study of the biophysical principles and vocabulary used in the field, to facilitate its study; (c) the role of complementary exams in the context of tutoring problems and (d) the development of teaching materials that use integrated content with a morphofunctional approach. Unique aspects, such as study difficulties related to knowledge of a foreign language and the use of social networks, and controversies regarding the teachers' role in the process and what it means to learn radiology in medical school were also considered elements that can be used as part of a modern approach, with radiology education currently being in a process of change. Conclusion: It was concluded, according to the perceptions of the students interviewed, that the morphofunctional curricular component often used in problem-based teaching might be used to teach radiology and imaging diagnosis, within a multidimensional, contextualized, and transdisciplinary perspective. This goes beyond its role as a means of providing complementary exams, significantly integrating other sources of knowledge (especially anatomy and pathology) and facilitating the learning of both radiology and related subjects addressed in each study module.

Aceito em: 25/9/18

\section{INTRODUÇÃO}

O ensino da radiologia no curso de medicina alcançou nova dimensão não só como instrumento de diagnóstico complementar, mas também por sua utilização como ferramenta de ensino, integrada ao estudo da anatomia e patologia, entre outros assuntos. Uma estratégia pedagógica adequada para a inserção da radiologia precisa valorizar o ponto de vista dos estudantes e reorganizar a estrutura curricular, de tal forma que permita a integração dos conteúdos com base nos princípios estruturais da interdisciplinaridade e transdisciplinaridade na educação ${ }^{1-13}$.
A interação da radiologia com outras disciplinas já vem sendo introduzida em muitas escolas médicas com o uso de metodologias ativas, alinhadas a concepções curriculares integradoras objetivando o desenvolvimento de competências voltadas para a assistência, gestão e educação em saúde $e^{3-9,11,14-18}$.

Nas escolas médicas que adotaram a aprendizagem baseada em problemas, surgiram dúvidas sobre como, o quê e quando introduzir a oportunidade de aprendizado da radiologia, despertando a necessidade de elaborar estratégias que tornem o ensino mais eficiente desde o primeiro semestre do curso médico ${ }^{19-22}$. 
Atualmente, estudos indicam que alunos de medicina, mesmo em seu primeiro ano, são capazes de construir conhecimentos sobre radiologia, o que enriquece sua compreensão a respeito dos processos fisiopatológicos, bem como da anatomia. Isto contraria a argumentação tradicionalista de que a radiologia é ensinada e aprendida com maior facilidade e consistência depois que os alunos têm exposição ao treinamento clínico, o que serve de referencial teórico para apoiar o estudo precoce da radiologia, no transcorrer do curso médico ${ }^{11,15,16}$.

Na maioria das escolas médicas no Brasil, o conjunto de competências operacionais e interpretativas em radiologia e diagnóstico por imagem não é obrigatório, a despeito do reconhecimento de sua importância. Todavia, por não haver um padrão estabelecido, a discussão a respeito da estratégia de ensino da radiologia durante o curso de medicina está aberta $^{23,24}$.

A escolha inadequada dos exames complementares de diagnóstico é ponto crítico da formação médica. A má utilização dos métodos de diagnóstico por imagem tem forte impacto negativo para o cuidado do paciente e onera o sistema de saúde, seja público ou privado. O desenvolvimento de uma estratégia vantajosa de ensino para a radiologia na graduação médica é importante no combate a essas inadequações 23,25 .

Em pesquisas sobre o ensino médico, a proposta de que mais tempo deveria ser dedicado à aprendizagem da radiologia é valorizada, indicando a necessidade de expandir a abordagem desse assunto, pois é a forma mais comum pela qual os médicos têm acesso à anatomia - normal e patológica - de seus pacientes, contribuindo para um diagnóstico correto, fundamental na prática clínica ${ }^{7,23}$.

Nenhuma estratégia para o ensino da radiologia no curso médico pode ser bem-sucedida se desconsiderar o estudante. Por isso, é necessário realizar pesquisas com a intenção de analisar o ponto de vista do aluno a esse respeito $3,5,7,9,14-16,20,26-29$.

O objetivo deste trabalho foi avaliar como estudantes de um curso de medicina percebem o ensino da radiologia, suas implicações e aplicações em medicina, especialmente no componente curricular morfofuncional, e identificam as características positivas e negativas, no processo de aprendizagem através do uso de metodologias ativas, para subsidiar a elaboração de estratégias adequadas para aperfeiçoar a construção do conhecimento.

\section{MÉTODO}

Estudo de natureza exploratória com abordagem qualitativa, por análise do discurso objetivando compreender o ensino da radiologia no componente curricular morfofuncional na percepção de alunos do curso de graduação em Medicina.
A amostra de alunos foi composta por amostragem intencional, pois houve uma escolha deliberada dos respondentes ${ }^{30}$. Os critérios de inclusão foram os seguintes: ser aluno do terceiro ou quinto semestres do curso de graduação em Medicina do Centro de Ciências Biológicas e da Saúde - Campus Belém-PA da Universidade do Estado do Pará regularmente matriculado; não ter graduação em outro curso superior na área de saúde, completa ou não. A inadequação a estes critérios foi considerada como único motivo de exclusão. Desse modo, os participantes foram selecionados de uma população de cem alunos. Não ter graduação em outro curso superior na área de saúde, seja completa ou incompleta, foi ponto central na seleção dos participantes, para afastar impressões referentes a experiências prévias em relação ao ensino da radiologia. De acordo com os critérios de inclusão, todos os alunos que aceitaram participar desta pesquisa assinaram o Termo de Consentimento Livre e Esclarecido, recebendo uma via desse documento, sendo que o presente estudo foi aprovado pelo comitê de ética CAEE 49390215.9.0000.0017.

Os participantes são apresentados no Quadro 1, que contém os dados de identificação pessoal (caracterização dos participantes da pesquisa) e dados complementares. A importância das informações complementares reside em fornecer subsídios para que a seleção dos participantes seja a mais heterogênea possível, dentro de uma amostragem intencional e deliberada, definida metodologicamente, em subgrupos segundo gênero, parentesco e semestre de curso.

A técnica de coleta de dados foi por meio de entrevista semiestruturada, norteada por perguntas com respostas abertas, que possibilita captar diferentes discursos, a fim de responder a questão norteadora definida nos objetivos do estudo. Todas as entrevistas foram gravadas e transcritas na íntegra. As entrevistas foram analisadas por meio da técnica de análise de conteúdo na modalidade análise temática, cujo eixo central é o tema objeto da pesquisa e consiste em descobrir os núcleos de sentido que compõe a comunicação, sendo composta de pré-análise, exploração do material, tratamento dos resultados obtidos e interpretação.

Após várias leituras das entrevistas foram separadas as frases ou palavras que constituíram as unidades de significado que estavam ligadas a questão norteadora do estudo. As unidades de significado foram organizadas em grupos de acordo com a relevância e semelhanças. Os dados das unidades que possuem temas semelhanças foram agrupados por categorização e o material submetido à análise temática, que, por sua vez, utiliza o "tema" como conceito central, para reunir em cada categoria as respostas semelhantes e pôde ser graficamente apresentado mediante uma frase. 
A construção do conhecimento pela abordagem qualitativa trouxe como resultado uma verdade contextualizada com base no sentido interpretado pela analise dos discursos onde a identificação dos temas, dos sentidos, dos significados e as implicações foram teorizados considerando os conteúdos semânticos ou latentes, coerentes, singulares e contraditórios ${ }^{30}$.

\begin{tabular}{|ccccc|}
\hline Entrevista & \multicolumn{5}{c}{$\begin{array}{c}\text { QuAdro 1 } \\
\text { Idade } \\
\text { (anos) }\end{array}$} & Gênero & $\begin{array}{c}\text { Semestre } \\
\text { do curso }\end{array}$ & $\begin{array}{c}\text { Parentesco com } \\
\text { médicos (pais, irmãos } \\
\text { ou avós médicos) }\end{array}$ \\
\hline 1 & 21 & Fem. & $3^{\circ}$ & Com irmão médico \\
\hline 2 & 30 & Masc. & $3^{\circ}$ & Sem parentes médicos \\
\hline 3 & 20 & Masc. & $5^{\circ}$ & Com pai médico \\
\hline 4 & 20 & Masc. & $3^{\circ}$ & Sem parentes médicos \\
\hline 5 & 19 & Masc. & $5^{\circ}$ & Sem parentes médicos \\
\hline 6 & 20 & Fem. & $5^{\circ}$ & Sem parentes médicos \\
\hline 7 & 18 & Fem. & $3^{\circ}$ & Sem parentes médicos \\
\hline 8 & 20 & Fem. & $5^{\circ}$ & Sem parentes médicos \\
\hline 9 & 20 & Fem. & $5^{\circ}$ & Sem parentes médicos \\
\hline 10 & 21 & Masc. & $5^{\circ}$ & Sem parentes médicos \\
\hline 11 & 19 & Masc. & $3^{\circ}$ & Com irmã médica \\
\hline 12 & 19 & Fem. & $3^{\circ}$ & Sem parentes médicos \\
\hline
\end{tabular}

\section{RESULTADOS E DISCUSSÃO}

A observação dos investigadores permitiu a identificação de conteúdos latentes e semânticos, em população estudada, composta por 12 alunos de medicina, sendo 06 terceiro semestre, 06 do quinto semestre, 03 masculinos e 03 femininos de cada semestre, com idades variando entre 18 e 30 anos.

Em relação ao conteúdo latente, não verbalizado de forma concreta, entendeu-se que os estudantes têm consciência do protagonismo discente. Entretanto, superestimam o papel do professor, não compreendendo sua posição como facilitador do processo de aprendizagem em um currículo que objetiva a aprendizagem baseada em problemas. É notório o pouco entendimento a respeito dos conceitos de interdisciplinaridade e transdisciplinaridade.

Os estudantes transpareceram que a atitude de docentes não alinhados com a aprendizagem baseada em problemas que, por defenderem a manutenção das paredes disciplinares, apontam como negativa a ideia de uma abordagem integrada e multidimensional dos temas estudados - tem gerado confusão e insegurança no alunado, o que torna óbvia a necessidade de que todos os participantes do processo de elaboração e execução de uma estratégia educacional estejam afinados.

No presente estudo, os entrevistados se mostraram inseguros quanto aos rumos que lhes são propostos no estudo da radiologia, pois percebem que a maioria dos professores não está preparada adequadamente para utilizar métodos de diagnóstico por imagem como ferramentas de ensino, muito menos orientá-los sobre o papel da radiologia como instrumento de diagnóstico complementar.

Em relação às questões da entrevista, são evidentes nas respostas quatro temas recorrentes, verbalizados com clareza, a respeito dos quais podemos extrair uma verdade contextualizada, subjetiva, porém determinante para a elaboração de uma estratégia vantajosa para a inserção da radiologia no ensino de graduação em Medicina com uso de metodologias ativas.

\section{- A necessidade de introdução precoce da radiologia}

E2) Eu já estou indo para a unidade de saúde, se eu já estou tratando com o paciente, às vezes, o paciente chega de retorno na unidade básica com uma radiografia, o professor te mostra a radiografia, te mostra a tomografia e se eu não tenho esse conhecimento aqui é assim, estou olhando, mas não sei o que estou olhando, eu não sei o que eu devo buscar.

E5) A introdução poderia acontecer já no primeiro ou no segundo semestre, logo no primeiro ano do curso que é sempre importante para entender ciências básicas, e nisso as imagens radiológicas podem ajudar na compreensão da anatomia [...].

E7) Quanto mais cedo se tem esse contato, mais fácil fica o aprendizado.

Considerando a relação entre ensino e serviço e o Sistema Único de Saúde (SUS) como escola, os meios de diagnóstico complementar devem ser trabalhados em uma abordagem multidimensional desde o início do curso médico de graduação, pois o contato do estudante com o paciente assim o requer.

Devido ao avanço tecnológico que permite explorar diversos aspectos anatômicos, histológicos, fisiológicos e patológicos, e em decorrência do fácil arquivamento das imagens digitalizadas, permitindo a formação de grandes coleções, é positiva a proposta de usar a radiologia como ferramenta de aprendizagem desde o inicio do curso. A percepção da necessidade de introdução precoce da radiologia no componente curricular do morfofuncional fica evidente nos relatos dos alunos ao afirmarem que isto serviria para introduzir conceitos básicos de radiologia de forma transversal nos módulos temáticos, possibilitando relacionar com conhecimentos das ciências básicas, como, por exemplo, anatomia. 


\section{- Problemas na aprendizagem dos princípios biofísi-} cos e vocabulário

E6) Esse vocabulário específico compromete um pouco até o interesse dos alunos pelo estudo, porque eles não conseguem ter uma compreensão boa dos textos e acabam se desmotivando pela disciplina que é importante.

E7) Eu já encontrei dificuldades porque eles utilizam siglas e outros vocabulários que talvez eu ainda no meu segundo ano ainda não conhecia, não tenha visto. O primeiro ponto é a utilização de siglas por eu não saber realmente o que aquilo deveria significar. A utilização de termos em inglês tambémé um obstáculo pra mim.

E10) Esses textos de radiologia utilizam nomenclaturas específicas, às vezes alguns termos que a gente tem que buscar, pesquisar separadamente o que significam. Além disso, muitas vezes os textos são, por exemplo, em inglês. Se soubéssemos como o exame é feito, como a imagem é formada, também ajudaria bastante [...].

O uso de textos de apoio para a construção do conhecimento não pode ser dificultado pela incompreensão dos termos do vocabulário técnico específico da radiologia, demonstrando a necessidade de trabalhar o vocabulário como prioridade.

O entendimento do processo de formação da imagem radiológica, bem como dos demais métodos de diagnóstico por imagem é valorizado pelos estudantes como facilitador do aprendizado.

A incompreensão do vocabulário pode também ser um fator a desestimular o aluno, conforme relatado na sexta entrevista.

\section{- Necessidade de material didático personalizado}

E3) Temos um atlas de anatomia, nós temos um livro-texto de anatomia [...] por mais que o livro-texto tente trazer uma ou outra coisa da radiologia, é pouco, quase nada. Referências de radiologia tratam das peculiaridades da radiologia e não fazem uma integração com as demais.

E10) [...] e quando a gente vai estudar é um estudo compartimentalizado, primeiro você estuda o livro de anatomia, depois histologia, depois radiologia e outras fontes.

E12) Quando a gente vê tudo ao mesmo tempo, junto, facilita mais a nossa compreensão do assunto, pena que não se tenha um material integrado pra estudar.
A literatura disponível é, em geral, disciplinar, o que acaba prejudicando a integração dos conhecimentos. O estudante parece entender que é menos trabalhoso desmontar a integração e estudar por partes ao invés de desenvolver a coligação dos temas, embora alguns atribuam valor ao estudo integrado.

Como o processo de aprendizagem esbarra na falta de material adequado para apoiar a proposta de ensino integrado, o estudante busca como alternativa ao estudo particular o ensino entre pares, usando até mesmo redes sociais, embora temeroso de que as informações ali veiculadas por outros estudantes sejam incorretas, o que o faz desejoso de que professores possam intervir por meio dessa tecnologia.

\section{- Necessidade de fortalecer a ligação do componente curricular morfofuncional com a tutoria}

E4) Uma das minhas críticas com relação à tutoria é que ela poderia incrementar o diagnóstico por imagem nos seus problemas pra nos ajudar a desenvolver ainda mais esse problema e termos mais conhecimento para fechamento.

E9) Eu acredito que, durante as próprias tutorias, poderia ter imagem de apoio nos problemas, poderia ter essa questão das imagens radiológicas pra quando a gente tiver acesso ao problema já também ter acesso às imagens e que essas coisas possam se complementar pra gente melhorar o processo de debate na tutoria mesmo e da forma como está sendo realizada aqui no morfofuncional, de ter essas imagens nos roteiros, pra gente poder identificar o normal e as doenças, quais são os correlatos radiológicos para determinadas doenças que estamos vendo no morfofuncional e na tutoria também.

E12) Na tutoria, mesmo que eles não coloquem imagem, eles podem colocar o resultado escrito dos exames. Eles falaram no exame que a menina estava com pneumotórax ou eles colocam alguma descrição da imagem e às vezes eles não dizem o que significa. Então, quando a gente tem esse apoio aqui no morfofuncional, a gente já consegue ter uma ideia melhor.

A exploração da radiologia na tutoria é insuficiente na abordagem dos princípios introdutórios, o que dificultará o entendimento da inserção precoce e integrada da radiologia com a finalidade de enriquecer a compreensão dos processos patológicos e alterações morfoestruturais por ocasião das atividades desenvolvidas no laboratório morfofuncional.

Aponta-se a necessidade de fortalecer o entendimento dos conceitos de interdisciplinaridade e transdisciplinaridade 
no aluno, bem como a ligação do componente curricular morfofuncional com a tutoria e habilidades clínicas.

Os estudantes exteriorizaram repetidas vezes a ideia errônea de que aprender radiologia na graduação em medicina tem relação com dar diagnóstico radiológico.

As indicações e contraindicações dos diversos métodos de imagem, bem como a reflexão a respeito de quais exames são apropriados à solução de cada problema não estão postas nas entrevistas como algo relevante na experiência dos estudantes em relação às tutorias.

Quanto à ligação do componente curricular morfofuncional com a tutoria, na percepção dos entrevistados, não há entrosamento entre esses dois componentes curriculares, pois diversos tutores não têm preparo adequado para tratar de temas da radiologia.

Ressaltam a importância do aprendizado acerca da radiologia e diagnóstico por imagem no componente morfofuncional e apontam que, se houvesse maior articulação entre esses dois componentes curriculares, o resultado seria o aumento da aprendizagem com a melhoria do raciocínio clínico e, portanto, elevação da qualidade na formação médica.

A singularidade de algumas respostas permite atribuir valor específico a dois aspectos verbalizados durante as entrevistas. O primeiro refere-se ao fato de que diversos termos usados em radiologia em língua portuguesa não encontram paralelo literal em língua estrangeira, especialmente na língua inglesa, sendo proposta a introdução da terminologia em idioma estrangeiro quando da abordagem do vocabulário próprio da radiologia. O segundo está relacionado ao uso de redes sociais na educação entre pares, como a utilização de grupos nos aplicativos para smartphones, como o whatsapp, para discutir imagens radiológicas ou tirar dúvidas. $\mathrm{O}$ fator negativo de que tais trocas de informações podem propagar erros foi referido, porém isso pode ser minimizado com o embasamento em fontes confiáveis de pesquisa, enriquecendo e trazendo credibilidade às informações.

Um aspecto controverso encontrado nas entrevistas reside na defesa da ideia de que um dos principais obstáculos à introdução bem-sucedida do diagnóstico por imagem enquanto ferramenta de aprendizagem seria a falta de professores especialistas na condução do processo de ensino, tema da fala dos estudantes em considerável número. Nesta série de entrevistas, algumas alusões a esse respeito foram:

E3) Eu acho que não é só o aluno que tem dificuldade com a radiologia, eu acho que muitos professores, não têm uma intimidade com esse ramo, Eu acho que, pra dar a aula e conseguir integrar a radiologia no laboratório morfofuncional, precisa ser radiologista.
E4) Claro que determinados especialistas e não especialistas, com certeza, se estudarem, se se dedicarem e tiverem bastante conhecimento daquele assunto, claro que eles vão ter capacidade de dar; mas o especialista por si só, por ter esse título de especialista, ele já é considerado capaz de dar determinado assunto.

E5) A respeito da questão do ensino da radiologia no morfofuncional, muitas das vezes a gente encontra problema de você não ter professores especialistas para dar tal matéria.

E6) Eu falo que professor de radiologia é fundamental porque, bem ou mal, muitos dos professores do morfofuncional não estão habituados com a prática das imagens radiológicas e não têm familiaridade ou, se têm, digamos assim, muito em relação a sua área somente; então, por isso digo da necessidade de um professor de radiologia, porque ele tem noção sobre todos os aparelhos, sobre os diversos sistemas, às vezes tem professores dentro do morfofuncional que não têm essa experiência. Talvez a capacitação desses professores pudesse torná-los competentes pra lecionar essa disciplina, no caso, que não é uma disciplina, mais esse eixo, digamos assim, que faz parte do morfofuncional.

E9) Um profissional especialista nesse assunto eu acredito que vai ser bem melhor, por ele ter mais conhecimento pra poder repassar; então eu acredito que esse conteúdo deve ser exposto por um profissional radiologista.

É importante salientar que a má compreensão dos estudantes a respeito da aprendizagem baseada em problemas e a dificuldade de desprendimento da visão disciplinar os levam à ideia de que seu rendimento seria maior se um professor especialista em radiodiagnóstico lhes fosse disponibilizado. Desta forma, podemos compreender que, por mais que um professor especialista não esteja disponível, é necessário que o docente, tanto na tutoria quanto no laboratório morfofuncional, transmita um mínimo de segurança ao estudante para que o processo de ensino-aprendizagem possa fluir. $\mathrm{O}$ aspecto provocativo do professor para com o estudante em relação aos objetivos do problema trabalhado requer consistência teórica mínima para que o docente cumpra seu papel facilitador da aprendizagem.

A defesa da necessidade de profissional especialista em radiologia como professor está ligada à falta de clareza do estudante em relação à aprendizagem baseada em problemas e à incompreensão da proposta pedagógica, vista no uso de expressões como "conhecimento pra poder repassar" ou "expor 
o conteúdo". Isto porque a ideia de construção e não de repasse do conhecimento, bem como o entendimento de que o papel do professor é o de facilitador e não de instrumento de "expositor do conteúdo" não estão incorporados ao pensamento dos estudantes entrevistados em relação a sua experiência educacional em instituição de nível superior, com a provável replicação do padrão mental transposto do ensino médio.

Outra questão controversa é o pensamento de que o ensino da radiologia na graduação em medicina deve enfatizar o treinamento na interpretação de imagens, em contraste com a ideia de valorizar a habilidade do aluno para solicitar exames adequados às diferentes situações clínicas e conhecer os sinais elementares, bem como entender a nomenclatura radiológica para que lhe seja possível compreender os laudos radiológicos.

E2) Os médicos têm buscado fechar seu diagnóstico só com base em exames, talvez por medo de errar, de dar um diagnóstico impreciso; então, pra mim, é essencial que o médico saiba disso (interpretar exames radiológicos).

E3) Esse tipo de coisa (radiografias) a gente só entende se a gente tem um conhecimento da física por trás da realização do exame, e a primeira olhada é bem difícil para o aluno eu acho, é algo que a gente só consegue na prática, com bastante exercício.

E4) Eu creio que seria mais prático pra todos os alunos ver como é uma situação radiológica normal, ver quais são as estruturas normais, pra depois ver o que está diferente, anormal; essa seria a minha sugestão para o ensino da radiologia.

E7) Eu acredito que ela seja ensinada de forma complementar à clínica, ela vai auxiliar as nossas, como é que eu posso falar? por exemplo, a gente tem a clínica em si, o exame físico, anamnese, que são o básico, digamos; ela (radiologia) vai vir pra auxiliar mais, ela pode ser essencial.

E11) Principalmente no serviço público, em que o diagnóstico laboratorial demora e às vezes não tem o radiologista, saber diagnóstico por imagem passa a ser de extrema importância. Ele vai definir muitas vezes o diagnóstico.

Enquanto alguns entendem que o fundamental é saber interpretar exames, outros enfatizam como aspecto central a capacidade de solicitá-los adequadamente. Observa-se também a preocupação em saber avaliar imagens e assumir a condição de examinador por questões conjunturais do sistema de saúde, quando o apoio de especialistas não estiver disponível.
A preocupação com a construção da capacidade para atuar na esfera das urgências e emergências no serviço público de saúde, demonstrada nas entrevistas, e a consideração feita sobre a necessidade de saber diagnosticar em exames de imagens os sinais radiológicos clássicos de situações clínicas graves são pertinentes, encontrando sintonia com artigos da literatura médica ${ }^{16}$.

Embora desejável, construir a habilidade em perceber anormalidades radiológicas obscuras requer treinamento de longo prazo, necessitando de tempo indisponível durante a graduação em medicina. Todavia, o treinamento para a identificação de anormalidades óbvias no contexto da urgência e emergência é exequível.

Os temas recorrentes, aspectos singulares e controvérsias, as suas relações e implicações estão exibidos no Quadro 2.

\section{QuAdro 2}

\section{Temas recorrentes, singulares e controvérsias,} suas relações e implicações

Temas recorrentes

- Necessidade de introdução precoce.

- Relacionada com a inserção do estudante no contexto do serviço de saúde.

- Problemas referentes a princípios biofísicos e vocabulário. - Prejudicam o entendimento da leitura de textos de apoio.

- Necessidade de material didático personalizado. - A literatura disponível é em geral de cunho disciplinar.

- Necessidade de fortalecer a ligação do componente curricular morfofuncional com a tutoria.

- No que diz respeito ao ensino da Radiologia, observa-se desarticulação entre as abordagens dos componentes curriculares.

\section{Singularidades}

- Abordagem em língua estrangeira.

- Relacionada com a necessidade de aumentar a compreensão dos textos de apoio, pois diversas nomenclaturas estão em língua estrangeira, usando expressões que não têm tradução literal em língua portuguesa.

- Uso de redes sociais.

- Relacionado ao ensino entre pares.

\section{Controvérsias}

- Necessidade de professor especialista em radiodiagnóstico para o ensino da Radiologia.

- Implicada na má compreensão do estudante a respeito do papel docente na aprendizagem baseada em problema; todavia, medida necessária caso a estratégia de ensino contemple medidas heterodoxas, como a utilização de aulas expositivas ou discussão de casos radiológicos.

- Entendimento de que o aprendizado da Radiologia na graduação em Medicina seja centrado no treinamento para a interpretação de imagens.

- A habilidade em perceber anormalidades radiológicas obscuras requer treinamento de longo prazo, necessitando de tempo indisponível durante a graduação em Medicina. 
Entre os temas recorrentes, na percepção dos estudantes, a necessidade de introdução precoce relacionada com a inserção do aluno no contexto do serviço de saúde é aspecto relevante entre os resultados desta pesquisa. Trata-se de um argumento significativo, por não ser apontado pelos autores que defendem a implementação do estudo do radiodiagnóstico desde o início do curso médico com base na associação com o aprendizado de anatomia e patologia ${ }^{1,9,15}$.

Os estudantes de medicina mencionaram insatisfação com: (I) a incompreensão dos princípios biofísicos; (II) a incompreensão de vocabulário específico da radiologia e demais métodos de diagnóstico por imagem, que foram apontados como prejudiciais ao entendimento da leitura de textos de apoio; (III) a dificuldades na utilização de textos em língua inglesa por falta de domínio do idioma; (IV) a falta de material didático personalizado, considerando-se que a literatura disponível é em geral de cunho disciplinar, corroborando a necessidade de elaborar-se uma estratégia de ensino que contemple tais demandas, como estratégias de $e$-Learning, já bem avaliadas na Europa e América do Norte ${ }^{31-34}$.

É notório nas entrevistas realizadas que, por mais que o professor em seu papel facilitador oriente o caminho aos objetivos de forma integrada e multidimensional, o aluno, por ocasião do estudo pessoal ou coletivo, recorrerá a fontes que promovem a abordagem disciplinar do que está sendo estudado. Devido ao emprego de abordagens de caráter indutivista-dedutivista, calcadas em episteme de análise (quebra e repartição), o estudante acaba por fragmentar a abordagem dos objetivos, o que deve ser evitado por meio de uma intervenção firme da equipe de professores ${ }^{42,43}$.

Elementos lúdicos, disponíveis em aplicativos de smartphones e tablets, além de canais ligados a tecnologias de comunicação e informação como o youtube, são bastante utilizados e defendidos por vários autores e pelos estudantes entrevistados. Nota-se crescimento do uso destes instrumentos como ferramenta didático-pedagógica, trazendo contribuição para a prática escolar em qualquer nível de ensino e área do conhecimento. No entanto, isso impõe mudanças nos métodos de trabalho dos professores, gerando modificações no funcionamento dos espaços de ensino $3,4,6,7,9,11,22,25,27,43,45,47-49$.

Estratégias lúdicas, transdisciplinares, interativas, implantadas desde o primeiro semestre do curso médico, para promover o ensino da radiologia no contexto da aprendizagem baseada em problemas, podem ser construídas a partir da contribuição dos próprios estudantes, cuja opinião deve ser valorizada em um ambiente acadêmico respeitoso do protagonismo do aluno ${ }^{25}$.
Os alunos são atrelados à visão disciplinar, o que torna difícil entender as propostas estratégicas que eliminam as paredes disciplinares.

\section{CONSIDERAÇÕES FINAIS}

Os estudantes de medicina da amostra estudada percebem que o componente curricular morfofuncional, na aprendizagem baseada em problemas, poderá ser usado para abordar a radiologia e diagnóstico por imagem numa visão multidimensional que vá além do papel de método de exame complementar, integrado a outros saberes, especialmente anatomia e patologia, facilitando a aprendizagem tanto da radiologia quanto dos demais assuntos envolvidos na temática abordada em cada módulo de estudo.

A carga horária destinada ao componente curricular morfofuncional pode ser usada para potencializar o estudo participativo, interpares, em pequenos grupos e multidimensional de temáticas relacionadas aos métodos de imagem, desde que venham atender ao fortalecimento do fechamento dos problemas na sessão tutorial, incluindo estudos de casos radiológicos, ferramentas lúdicas como jogos de perguntas, pequenas aulas expositivas, estudos de textos, simulações em computadores, busca de informações em bases de dados científicas e aprendizagem baseada em equipes ${ }^{25,33-50}$. Espera-se que, as atividades propostas no componente morfofuncional através de roteiros com objetivos estruturados de aprendizagem desenvolvam, no estudante, habilidades para síntese do conhecimento através de fluxograma, mapa conceitual e outras representações gráficas, administração do tempo, trabalho em equipe e visão multidisciplinar na resolução de problemas ${ }^{33-50}$.

Fortalecer a ligação do componente curricular morfofuncional com a tutoria é importante para o processo de ensino-aprendizado da radiologia. Por isso, o planejamento das atividades docentes e o diálogo entre tutores e os que atuam em outros componentes devem ser intensificados. Muitas vezes, a desarticulação entre a abordagem feita em diferentes componentes curriculares é apontada como entrave ao bom andamento de cursos médicos que se valem da aprendizagem baseada em problemas, perdendo-se a oportunidade de integração $^{44,48}$.

A acurácia diagnóstica em radiologia não é habilidade fundamental ao estudante de Medicina, mas, sim, o domínio do vocabulário técnico para o entendimento dos laudos dos exames solicitados, bem como a utilização adequada dos métodos de diagnóstico por imagem. Essas competências podem ser desenvolvidas dentro dos diversos componentes curriculares da aprendizagem baseada em problemas, especialmente nas habilidades clínicas ${ }^{50}$. 
As atividades do laboratório morfofuncional podem ser incrementadas com a utilização de táticas heterodoxas sob a perspectiva da aprendizagem baseada em problemas, porém úteis para introduzir a radiologia e outros métodos de diagnóstico por imagem, como a aula temática expositiva e dialogada, a aprendizagem baseada em equipes, o estudo de textos e o estudo de casos radiológicos, partindo de uma visão estratégica apoiada sobre os pilares do ensino precoce, transdisciplinar, interativo e lúdico, compatibilizados com os módulos temáticos explorados nas tutorias, com apoio colateral das habilidades clínicas.

O presente estudo foi realizado em apenas uma instituição, além disso, por se tratar de pesquisa qualitativa tem um número reduzido de participantes, o que limita possíveis generalizações.

\section{REFERÊNCIAS}

1. Starkey D. Integration of medical images to the teaching of systematic pathology: an evaluation of relevance. Journal of Learning Design 2011; 4(3) 63-70.

2. Albarracín ES, Silva SCR, Schirlo AC. Interdisciplinaridade: saberes e práticas rumo à inovação educativa. Interciencia 2015; 40 (1) 63-67.

3. BRASIL. Ministério da Educação. Conselho Nacional de Educação. Câmara de Educação Superior. Resolução № 3, de 20 de junho de 2014. Institui Diretrizes nacionais do curso de graduação em medicina. Diário Oficial da União, Brasília, 23 jul. 2014; Seção 1, p. 8-11. 2014.

4. Chorney ET, Lewis PJ. Integrating a Radiology Curriculum Into Clinical Clerkships Using Case Oriented Radiology Education. Journal of the American College of Radiology 2011; 8 (1) 58-64.

5. Farias PAM, Martin ALAR, Cristo CS. Aprendizagem Ativa na Educação em Saúde: Percurso Histórico e Aplicações. Revista Brasileira de Educação Médica 2015; 39 (1) 143-58.

6. Dettmer S, Weidemann J, Fischer V, Wacker FK. Integrative Teaching in Radiology - A Survey. Fortschritte auf dem Gebiete der Röntgenstrahlen. 2015; 187(4) 260-68

7. Ekelund L, Elzubeir M. Diagnostic Radiology in an Integrated Curriculum: Evaluation of Student Appraisal. Academic Radiology 2000; 7 (11) 965-70

8. Florentino JA, Rodrigues LP. Disciplinaridade, interdisciplinaridade e complexidade na educação: desafios à formação docente. Educação Por Escrito [online]. 2015. 6 (1) [capturado 4 jan. 2017]; 54-67. Disponível em:http:// www.revistaseletronicas.pucrs.br/ojs/index.php/porescrito/article/download/17410/12794
9. Heptonstall NB, Ali T, Mankad K. Integrating radiology and anatomy teaching in medical education in the UKThe evidence, current trends, and future scope. Academic Radiology 2016; 23 (4) 521-26.

10. Gunderman RB, Stephens CD. Teaching medical students about imaging techniques. AJR American Journal of Roentgenology 2009; 192 (4) 859-61.

11. Kourdioukova EV, Valcke M, Derese A, Verstraete KL. Analysis of radiology education in undergraduate medical doctors training in Europe. European Journal of Radiology 2011; 78(3) 309-18.

12. Hunter D J, Frenk J. The Birth of Public Health Education. JAMA 2015; 313 (11) 1105-106.

13. Martins JJN, Rego S, Lampert JB, Araújo JGC. Educação médica em transformação: instrumentos para a construção de novas realidades. São Paulo: Hucitec. 2004.

14. Miles K A. Diagnostic imaging in undergraduate medical education: an expanding role. Clinical Radiology 2005; 60 (7) $742-45$.

15. Branstetter BF, Faix LE, Humphrey AL, Schumann JB. Preclinical Medical Student Training in Radiology: The Effect of Early Exposure. AJR American Journal of Roentgenology 2007; 188 (1) 9-14.

16. Kalami TR, Hood A, Craven I. Undergraduate radiology teaching: starting from scratch. Clinical Radiology 2016; 71 (S1) 5-6.

17. Moraes MC, Navas JMB. Complexidade e transdisciplinaridade em educação: teoria e prática docente. Rio de Janeiro: Wak. 2010.

18. Pagliosa FL, Ros MA. O relatório Flexner: para o bem e para o mal. Revista Brasileira de Educação Médica 2008; 32(4) 492-99.

19. Keats TE. Medical education in radiology in Sweden.Radiology 1965; 84 (3) 547-53.

20. Martins JJN, Rego S, Lampert JB, Araújo JGC. Educação médica em transformação: instrumentos para a construção de novas realidades. São Paulo: Hucitec. 2004.

21. Salmon M, Williams D, Rhee K. Refocusing Medical Education Reform: Beyond the How. Academic Medicine 2015; 90(2) 136-38.

22. Souza CS, Iglesias A G, Pazin-Filho A. Estratégias inovadoras para métodos de ensino tradicionais - aspectos gerais. Medicina (Ribeirão Preto) 2014; 47 (3) 284-92.

23. Souza AMV, Barbosa FTRG, Messias RB, Neto JFR, Araújo LM, Souza e Souza LP, Brito MFSF, Santos SP, Reis TC. O ensino da radiologia na graduação médica. Revista Norte Mineira de Enfermagem 2014; 3(2) 64-78. 
24. McDonald KM, Matesic B, Contopoulos-IoannidisD G, Lonhart J, Shimidt E, Pineda N, Ioannidis JPA. Patient safety strategies targeted at diagnostic errors. Annals of Internal Medicine 2013; 158 (5) 381-89.

25. Silva AF, Freiras JJS, Domingues RJS. Ensino da radiologia com uso de metodologias ativas na graduação em medicina. Revista Interdisciplinar de Estudos em Saúde 2016; 5 (2) 41-56.

26. Larocque N, Kenny S, McInnes MDF. Medical school radiology lectures: What are determinants of lecture satisfaction? AJR American Journal of Roentgenology 2015; 204 (5) 913-18.

27. Watmough S, Cherry MG, O'Sullivan H. A comparison of self-perceived competencies of traditional and reformed curriculum graduates 6 years after graduation. Medical Teacher 2012; 34 (7) 562-68.

28. Willianson KB, Gunderman RB, Cohen MD, Frank MS. Learning theory in radiology education. Radiology 2004; 233 (1) $15-18$.

29. Ghasemizad A. Learning strategies and academic success in traditional and nontraditional higher education students. International Journal of Education and Psychological Researches 2015; 1 (1) 7-9.

30. Turato ER. Tratado de metodologia da pesquisa clínico-qualitativa: construção teórico-epistemológica, discussão comparada e aplicação nas áreas da saúde e humanas. 5.ed. Petrópolis: Vozes, 2011.

31. Zafar S, Safdar S, Zafar AN. Evaluation of use of e-Learning in undergraduate radiology education: A review. European Journal of Radiology 2014; 83 (12) 2277-287.

32. Webb AL, Choi S. Interactive radiological anatomy eLearning solution for first year medical students: Development, integration, and impact on learning. Anatomic Science Education 2014; 7 (5) 350-60.

33. Corl FM, Johnson PT, RowellmR, Fichman, EK. Internet-based dissemination of educational video presentations: A primer in video podcasting. AJR American Journal of Roentgenology 2008; 191(1) 23-27.

34. Dang AP, Kalra MK, Schultzt F, Grahams A, Dreyer KF. Informatics in radiology render: an online searchable radiology study repository. Radiographycs 2009; 29 (5) 1233-1246.

35. Gonzalez SM, Gadbury-Amyot CC. Using Twitter for teaching and learning in an oral and maxillofacial radiology course. Journal of dental education 2016; 80(2) 149-55.

36. Rowe SP, Siddiqui A, Bonekamp D. The key image and case log application: new radiology software for teaching file creation and case logging that incorporates elements of a social network. Academic Radiology 2014; 21(7) 916-30.
37. Costa J M, Araújo AT, Silva BM. A midiatização no processo de ensino e aprendizagem. Revista Tecnologias na Educação 2014; 11(11).1-11.

38. Geraldeli FE, Carvalho CP, Koch HA, Azevedo ACP. Produção de material instrucional para o ensino da radiologia por meio da digitalização de imagens. Radiologia Brasileira $2002 ; 35(1)$ 27-30.

39. Hoppe L, KroeffAMS. Educação Lúdica no Cenário do Ensino Superior. Revista Veras 2014; 4 (2) 164-81.

40. Coscrato G. Pina JC, Mello DF. Utilização de atividades lúdicas na educação em saúde: uma revisão integrativa da literatura. ActaPaulista de Enfermagem 2009; 23(2) 257-63.

41. Helle L, Säljö R. Collaborating with digital tools and peers in medical education: cases and simulations as interventions in learning. Instructional Science 2012; 40 (5) 737-44.

42. Telang A. Problem-based learning in health professions education: An overview. Archives of Medicine and Health Sciences 2014; 2 (2) 243-46.

43. Mello CCB, Alves RO, Lemos SMA. Metodologias de ensino e formação na área da saúde: revisão de literatura. Revista CEFAC 2014; 16(6) 2015-28.

44. Montes MAA, SOUZA, CTV. Estratégia de ensino-aprendizagem de anatomia humana para acadêmicos de medicina. Ciências e Cognição 2010; 15 (3) 2-12.

45. Abrahão AL, Merhy EE. Formação em saúde e micropolítica: sobre conceitos-ferramentas na prática de ensinar. Interface Botucatu 2014; 18 (49) 313-324.

46. Luiz LC, Oliveira LF, Batista RT. O uso de ilustrações no ensino e no setor de radiologia como uma proposta para construção dos conceitos de física radiológica e radioproteção. Revista Brasileira de Física Médica 2011;5(3) 245-52.

47. Nacif MS, Gomes AP, Santos SS; Freita LO, Medeiros-Souza RR, Siqueira-Batista R, Ensino e pesquisa em radiologia e diagnóstico por imagem no curso de graduação em medicina: uma perspectiva interdisciplinar. Revista Ciência e Ideias 2011; 3 (1) 1-9.

48. Silva AF. Estratégia para a inserção da radiologia no ensino de graduação em medicina com uso de metodologias ativas. Belém-Pará; 2017. Mestrado ensino em saúde na Amazônia [Dissertação] - Universidade do Estado do Pará.

49. Zou L, King A, Soman S, Lischuk A, Schneider B, Walor D, Bramwit M, Amorosa JK. Medical students' preferences in radiology education a comparison between the Socratic and didactic methods utilizing powerpoint features in radiology education. Academic Radiology 2011; 18 (2) 25356.

50. Guderman RB, Nyce JM. The tyranny of accuracy in radiology education. Radiology 2002; 222 (2) 297-300. 


\section{CONTRIBUIÇÃO DOS AUTORES}

Todos os autores participaram da concepção, planejamento e produção do artigo.

\section{CONFLITO DE INTERESSES}

Não há conflito de interesse de nenhuma natureza.

\section{ENDEREÇO PARA CORRESPONDÊNCIA}

Robson José de Souza Domingues

Trav. Perebebuí, 2623, Bairro do Marco Belém, Pará, BR 66.087-670 - domingues@uepa.br 